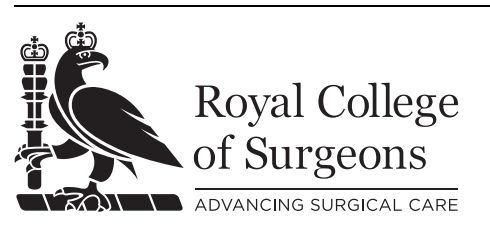

\title{
ENDOCRINE SURGERY
}

\section{Association between thyroid nodule size and malignancy rate}

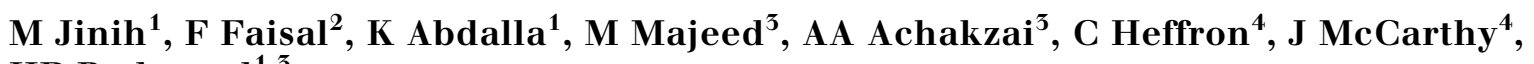 \\ HP Redmond ${ }^{1,3}$
}

${ }^{1}$ Department of Academic Surgery, Cork University Hospital, Wilton, Cork, Ireland

${ }^{2}$ School of Medicine, University College Cork, Cork, Ireland

${ }^{3}$ Department of ENDOCRINE SURGERY, Cork University Hospital, Wilton, Cork, Ireland

${ }^{4}$ Department of Pathology, Cork University Hospital, Wilton, Cork, Ireland

\section{ABSTRACT}

INTRODUCTION The diagnostic performance of ultrasound-fine needle aspiration to identify thyroid nodules harbouring malignancy remains variable. The aim of this study was to determine thyroid nodule size and cytological classification as predictors of malignancy risk.

MATERIALS AND METHODS We conducted a retrospective cohort analysis at an academic hospital involving 499 consecutive patients who underwent thyroid surgery between 2004 and 2015.

RESULTS A total of 503 thyroid nodules (499 patients, 84\% female; mean age 50.8 years, standard deviation, SD, 15.4 years) were analysed. Of these, $19.5 \%$ were malignant. The mean $( \pm$ SD) nodule size was $3.28 \pm 1.63 \mathrm{~cm}$ and $3.27 \pm 1.54 \mathrm{~cm}$ for benign and malignant nodules, respectively. The odds of malignancy for thyroid nodules less than $3.0 \mathrm{~cm}$ was similar to those for nodules of $3.0 \mathrm{~cm}$ or greater $(0.26$ compared with $0.29 ; p=0.77)$. Overall, the sensitivity and specificity of fine-needle aspiration in this cohort were $71.4 \%$ and $100 \%$, respectively. The overall false negative rate was $5.4 \%$. When the cut-off of $3.0 \mathrm{~cm}$ was used, the false negative rate in thyroid nodules less than $3.0 \mathrm{~cm}$ was $0 \%$ compared with $7.0 \%$ in nodules of $3.0 \mathrm{~cm}$ or greater. Thus, class $(p<0.01)$ but not nodule size $(p=0.49)$, was associated with higher malignancy risk.

CONCLUSIONS Our results suggest that thyroid nodule size did not accurately predict the risk of thyroid malignancy irrespective of fine-needle aspiration cytology. Routine diagnostic thyroid lobectomy solely owing to thyroid nodule size of $3.0 \mathrm{~cm}$ or greater is currently not justified.

\section{KEYWORDS}

Thyroid nodule - Malignancy - Cancer

Accepted 8 September 2019

CORRESPONDENCE TO

Marcel Jinih, E: marceljinih@yahoo.com

\section{Introduction}

Thyroid cancer is the most common form of endocrine malignancy and recent data indicate that it is currently the fifth most common cancer worldwide., ${ }^{1,2}$ Although most thyroid nodules are benign, ${ }^{3}$ approximately $5-15 \%$ turn out to be malignant on final histopathology. ${ }^{4}$ Furthermore, most thyroid cancer manifests as thyroid nodules with or without any associated symptoms. ${ }^{5}$ Unlike other cancers that show stable or declining trends, the incidence of thyroid cancer has continued to surge globally over the past few decades. ${ }^{6}$ The incidence in the United States, for example, has increased dramatically to $4.5 \%$, while in Great Britain the incidence has risen at $3.4 \%$ in both genders. $^{2,7}$
Ultrasound-guided fine needle aspiration is an important preoperative diagnostic tool to identify thyroid nodules harbouring malignancy. ${ }^{8}$ Data indicate that the false-negative rate of fine-needle aspiration in detecting thyroid cancer is $0-5 \% .{ }^{9}$ However, when the false negative rate is stratified according to nodular size, conflicting results emerge. Many studies reported that large nodule size $(\geq 3.0 \mathrm{~cm})$ carries a higher false negative rate, ranging from $10.4 \%$ to as high as $25 \% .{ }^{10-15}$ Consequently, many clinicians advocated that diagnostic thyroid lobectomy be performed based solely on nodule size. ${ }^{13,14,16}$ In contrast, other studies have found that nodule size did not adversely affect the false negative rates of fine-needle aspiration. ${ }^{17-24}$ In this study, we sought to determine and evaluate the diagnostic accuracy and predictive values of ultrasound-guided fine-needle 
aspiration in our institution, specifically looking at the nodule size and cytology.

\section{Materials and methods}

\section{Data collection}

Approval was obtained from the Clinical Research Ethics Committee of the Cork Teaching Hospitals prior to the commencement of this study. Data were retrospectively collected from prospectively maintained theatre logbooks on patients who underwent thyroid surgery in Cork University Hospital by a single endocrine surgeon between January 2004 and December 2015. The inclusion criteria were: (a) patients who underwent first time thyroid surgery for thyroid nodules; (b) patients with complete preoperative investigations performed; and (c) patients with complete final histopathology reports after the surgery. Patients were excluded if: (a) the surgery was a repeat surgery (eg completion thyroidectomy); (b) there were incomplete preoperative investigation reports; and (c) incomplete final histopathology surgery after the surgery.

In our institution, all thyroid nodules subjected to ultrasound-guided fine-needle aspiration and cytology evaluation are discussed in a multidisciplinary meeting between the radiologists, pathologists, endocrine surgeons/ physicians and oncologists/radiotherapists. The criteria for surgery includes cytology report of malignancy (Thy 5); suspected malignancy (Thy 4); concern for follicular neoplasm (Thy 3f), undetermined with atypia (Thy 3a) in at least two biopsies performed at different times; benign cytology (Thy 2) but large-sized nodules $(\geq 2.0-3.0 \mathrm{~cm})$; benign cytology result but with high suspicions of malignancy in ultrasonographic and clinical examinations; concurrent thyroidectomy made during excision of parathyroid adenoma; and patient's concern.

The following data were collected: patient's initials and medical chart number, sex, date of birth, age, date and type of surgery, preoperative investigations and final histopathology reports. For preoperative investigations, information such as cytology classification based on the British Thyroid Association classification was collected and includes the primary side and size of the dominant nodules, and the corresponding final histopathology diagnosis. ${ }^{9}$ In cases of repeated fine-needle aspiration cytology for a single thyroid nodule, the highest cytology classification was taken. Only the dominant thyroid nodule was recorded based on the ultrasound report of the largest dimension in centimetres. Following surgery, the corresponding histopathology reports of the nodules were recorded. Because our institution only adopted the standardised fine-needle aspiration reporting system from year 2011 onwards, only data from this period was used to calculate false negative rate, sensitivity and specificity.

\section{Statistical analyses}

The main outcome parameters were the probability of thyroid malignancy as a function of nodule size and cytology classification. Fine-needle aspiration sensitivity, specificity and positive likelihood ratio were calculated according to cytology classification with the corresponding 95\% confidence interval (CI). To calculate these, Thy 2 (Bethesda II) cytological finding was considered as a negative result while Thy 4 to Thy 5 (Bethesda V-VI) were considered as positive test results for malignancy. Thy 1 , Thy3a and Thy3f (Bethesda I, III and IV) were included in the summary tables but excluded in calculations involving fine-needle aspiration false-negative rate, sensitivity and specificity. The false negative rate was defined as thyroid nodules being reported as benign under fine-needle aspiration cytology but malignant on final histopathological examination after surgery. Surgical histopathology results represent the gold standard in determining malignancy in any given thyroid nodule. Diagnostic odds ratio was also calculated as a single indicator measure of the diagnostic test accuracy.

The probability of malignancy as a function of nodule size and fine-needle aspiration cytology was modelled using two models of binomial logistic regression analysis. The first model evaluated the probability of malignancy as a function of nodule size alone $(n=503)$. The second model evaluated the probability of malignancy as a function of both nodule size and fine-needle aspiration cytology ( $n=187)$. Results of the binomial logistic regression models were reported using predicted probabilities. A two-sided $p$-value of less than 0.05 was considered as statistically significant. Statistical analysis was performed using IBM SPSS version 23 and Graphpad Prism version 6.

\section{Results}

\section{Patient demographics}

During the period from January 2004 to December 2015, 754 patients underwent thyroid surgery for various reasons. Of these, 499 patients involving 503 thyroid nodules were included in the study because they met our inclusion criteria. Our study sample comprised 419 (84\%) women and $80(16 \%)$ men. The mean age of the overall sample was 50.74 years (SD 15.41) (95\% CI 49.39-52.09) and ages ranged between 14 years and 99 years (Table 1). There was no significant difference in mean age between female and male patients (50.44 vs 52.84).

\section{Histopathology and fine-needle aspiration test characteristics}

The mean size of thyroid nodule $( \pm$ SD) was $3.275 \mathrm{~cm}( \pm$ $1.61 \mathrm{~cm}$ ) with a $95 \%$ confidence interval of 3.13-3.42. Cytological breakdown of the thyroid nodules and mean nodule size are summarised in Table 1 and Figure 1. When a reproducible classification of fine-needle aspiration cytology reporting was adopted into our institution in 2011, only thyroid nodules from this period was analysed for cytology and fine-needle aspiration test characteristics $(n=187)$. In this cohort, 30 of $187(16 \%)$ of the nodules were malignant on final surgical histopathology. The rates of malignant disease according to fine-needle aspiration cytological classification were $0 \%$, $5.4 \%, 15.4 \%, 22.8 \%, 100 \%$ and $100 \%$ for patients in British 
JINIH FAISAL ABDALLA MAJEED ACHAKZAI HEFFRON MCCARTHY REDMOND
ASSOCIATION BETWEEN THYROID NODULE SIZE AND MALIGNANCY RATE

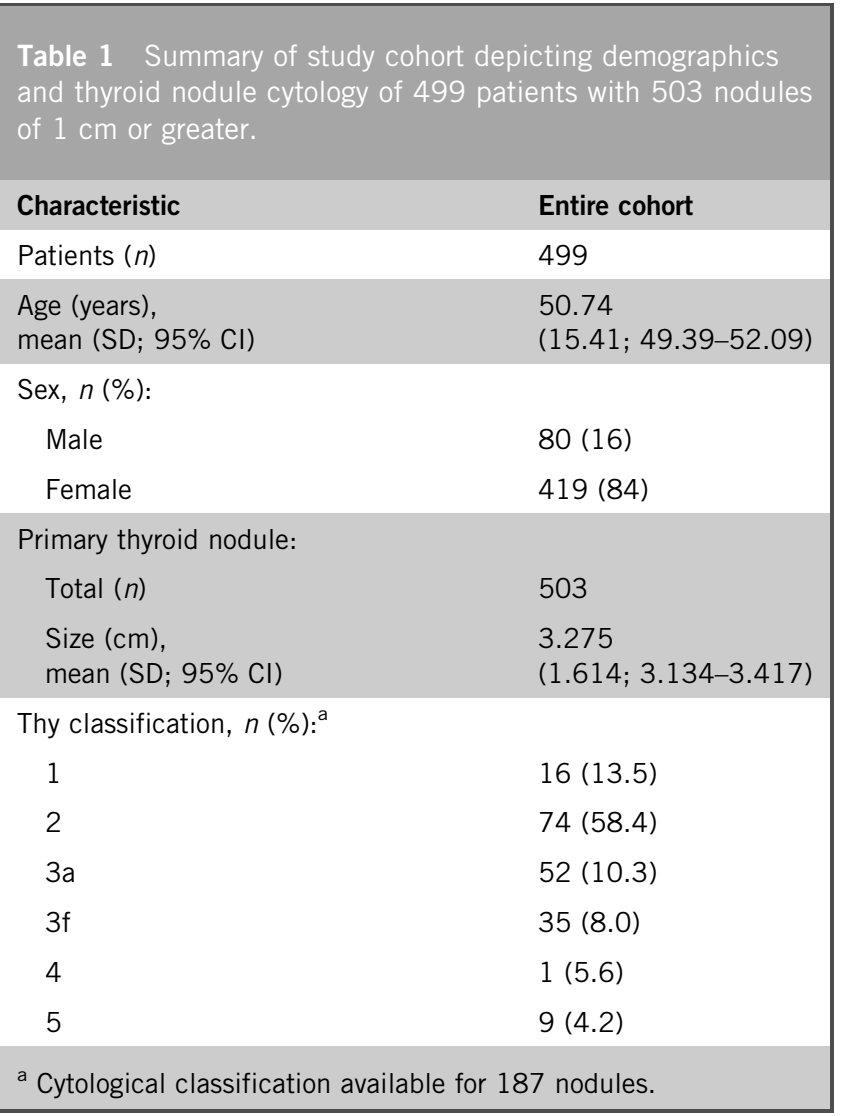

Thyroid Association classes Thy 1 to Thy 5 (corresponding Bethesda classes I to VI). Histologically, classic papillary thyroid carcinoma was the most common type of malignancy in this cohort $(56.7 \%)$ followed by follicular variant of papillary thyroid carcinoma at $23.3 \%$ and follicular thyroid carcinoma at $13.3 \%$ (Table 2). After excluding for Thy1, Thy3a and Thy3f (Bethesda I, III and IV), a total of 84 nodules were evaluated. Overall, the sensitivity and specificity of fine-needle aspiration in this cohort were $71.4 \%(95 \%$ CI $41-92 \%)$ and $100 \%$ (95\% CI $95-100 \%$ ), respectively. The overall false negative rate was $5.4 \%$. When the cut-off of $3.0 \mathrm{~cm}$ was used, the false negative rate in nodules less than $3.0 \mathrm{~cm}$ was $0 \%$ compared with $7.0 \%$ in nodules of $3.0 \mathrm{~cm}$ or greater.

\section{Nodule size and the rate of malignancy}

For the purpose of this study, the rates of malignancy were compared based on the nodule size. Overall, there was no significant difference in the rate of malignancy on subgroup analysis based on nodule sizes. The rates of malignancy according to every $1.0 \mathrm{~cm}$ increase in nodule size are summarised in Table 3 . When the $3.0 \mathrm{~cm}$ cut-off point was used, the rates of malignancy between nodules less than $3.0 \mathrm{~cm}$ compared with nodules of $3.0 \mathrm{~cm}$ or greater was not statistically different $(18.1 \%$ vs $20.6 \%$, $p=0.5$ ) and the odds of malignancy were similar for nodules less than $3.0 \mathrm{~cm}$ compared with nodules of 3.0 cm or greater $(0.264,95 \%$ CI $0.205-0.341$ vs $0.288,95 \%$ CI $0.168-0.494 ; p=0.77)$.

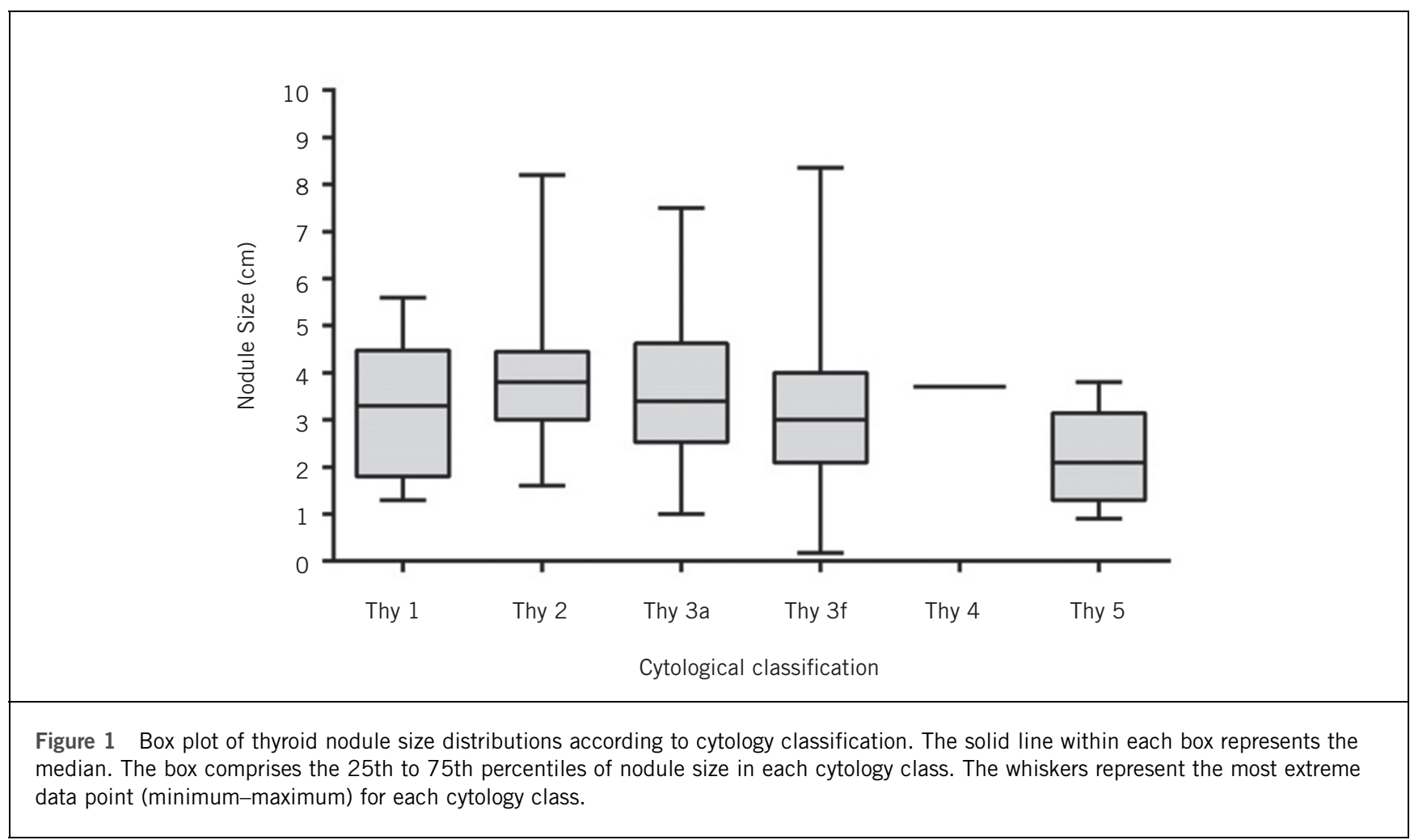




\begin{tabular}{|c|c|c|c|c|c|c|}
\hline \multirow[t]{2}{*}{ Characteristic } & \multicolumn{6}{|c|}{ Thy classification } \\
\hline & 1 & 2 & $3 a$ & $3 f$ & 4 & 5 \\
\hline Thyroid nodules, $n$ (malignant, $n$ ) & $16(0)$ & $74(4)$ & $52(8)$ & $35(8)$ & $1(1)$ & $9(9)$ \\
\hline Rate of malignant neoplasm (\%) & 0 & 5.4 & 15.4 & 22.8 & 100 & 100 \\
\hline \multicolumn{7}{|l|}{ Pathology of malignant nodules, $(n)$ : } \\
\hline Papillary thyroid cancer (PTC) & 0 & 3 & 3 & 2 & 0 & 9 \\
\hline PTC follicular variant & 0 & 1 & 2 & 3 & 1 & 0 \\
\hline Follicular thyroid cancer (FTC) & 0 & 0 & 2 & 2 & 0 & 0 \\
\hline FTC, Hürtle cell variant & 0 & 0 & 0 & 1 & 0 & 0 \\
\hline Medullary thyroid cancer & 0 & 0 & 1 & 0 & 0 & 0 \\
\hline
\end{tabular}

\begin{tabular}{lll|}
\hline $\begin{array}{l}\text { Table } 3 \\
\text { (every } 1 \mathrm{~cm} \text { increment). }\end{array}$ & Rate of thyroid nodule malignancy according to size \\
TN size $(\mathrm{cm})$ & Nodules $(\boldsymbol{n})$ & Malignant, $\mathbf{n}(\%)$ \\
$1.0-1.9$ & 90 & $17(18.9)$ \\
$2.0-2.9$ & 131 & $23(17.6)$ \\
$3.0-3.9$ & 136 & $27(19.9)$ \\
$4.0-4.9$ & 73 & $15(20.5)$ \\
$\geq 5.0$ & 73 & $16(21.9)$ \\
All nodules & 503 & $98(19.5)$ \\
\hline
\end{tabular}

Binomial logistical regression determining the probability of malignancy was performed based on two models: nodule size alone, and nodule size and FNA cytology. In the first model, nodule size $(n=503)$ was used in the logistic regression model. Actual nodule size was not predictive of thyroid malignancy in this model (OR $0.99, p=0.99$ ) with a Hosmer and Lemeshow test $p$-value of 0.25 . Similarly, not every $1 \mathrm{~cm}$ increase in nodule size (Table 3) was predictive of malignancy (OR 1.06, $p=0.49$ ), nor was the $3.0 \mathrm{~cm}$ cut-off point (OR $0.85, p=0.49$ ). In the second model, both nodule size and fine-needle aspiration cytology were evaluated as potential predictors of malignancy $(n=187)$. As expected, fine-needle aspiration cytology was predictive of malignancy (OR 3.57, $p<0.001$ ) but not the actual nodule size (OR 1.06, $p=0.67$ ).

\section{Discussion}

This study examines the association between thyroid nodule size and malignancy rate at a single institution. Evaluation of nodule size and the probability of malignancy remains an interesting area of research because, to date, there is no consensus as to whether nodule size does in fact predict thyroid malignancy. Our study aimed to add to the large body of information pertaining to the relationship between nodule size and the rate of malignancy and to elucidate whether or not there is a cut-off size for nodule resection. Some studies showed that large nodule size $(\geq$ $3.0-4.0 \mathrm{~cm}$ ) is associated with an increased probability of malignancy and have advocated surgical excision of these nodules. ${ }^{10,13,15,25}$ On the other hand, other studies have reported that the rate of malignancy does not increase substantially when comparing large and small nodules, and have advocated that surgery should not be performed on the basis of large nodule size alone. ${ }^{23,26-28}$ Another group reported that small nodule size is associated with a higher rate of malignancy up to a threshold of about 2.0-3.0 cm. ${ }^{22,24,29,30}$ Kamran et al, for example, reported that in small nodules $(1.0-2.0 \mathrm{~cm})$ the risk of malignancy rises proportionally up to a threshold of $2.0 \mathrm{~cm}$, beyond which there is no graded increase in malignancy. ${ }^{24}$ Additionally, Cavallo et al reported that the highest malignancy risk was observed in nodules of less than 2.0 $\mathrm{cm}$, with no graded decrease beyond the $2.0-\mathrm{cm}$ threshold. ${ }^{31}$

There are currently no clear guidelines as to what size warrants surgical excision, so the indications for surgery differ between institutions. Compared with previous studies, ${ }^{10,13,15,25}$ our data show that nodule size was not predictive of thyroid malignancy for every $1.0-\mathrm{cm}$ increment (Table 3) or when grouped into nodules of less than $3.0 \mathrm{~cm}$ compared with those of $3.0 \mathrm{~cm}$ or greater (malignancy rates of $18.1 \%$ vs $20.6 \%$ ). The results were similar when fine-needle aspiration cytology was factored into the analysis using a binomial logistic regression. Although large nodule size has been suggested as a possible risk factor for malignancy, there is very little evidence supporting this suggestion at the moment.

One of the major obstacles in studying the relationship between nodular size and the incidence of malignancy is that the final histopathological diagnoses are only available in nodules subjected to surgery. For the majority of nodules not subjected to surgery, the final histopathology is simply not available for analysis. The apparent difference in malignancy rates between the different nodule size groups as reported by previous studies may be explained 
by several factors. First, smaller nodules are usually selected for surgery only if there is a suspicion of malignancy either cytologically, radiologically or clinically. Therefore, only those with strong possibility of malignancy are undergoing surgery in this group. Second, compared with smaller nodules, large nodules $(\geq 3.0-4.0 \mathrm{~cm})$ are more likely to undergo surgery even if the cytology is benign. ${ }^{15}$ This is largely because of concerns of high malignancy rate, as reported by some earlier studies, ${ }^{10,15}$ and higher false negative rates. ${ }^{10,11,15,31}$ Finally, large nodules are more likely to cause clinical complaints of compression and cosmetic issues to the patients and are more likely to be excised, regardless of cytology or radiological appearances.

Another way of elucidating whether larger nodule size warrants surgery or not is to evaluate and compare the false negative rates between the different sizes. A number of studies have examined this question and the results have been variable. Several studies showed that a nodule size of $3.0 \mathrm{~cm}$ or greater is associated with higher false negative rates compared with a nodule size of less than 3.0 $\mathrm{cm}$, ranging between $5.8 \%$ to $17 \% .^{10,12,14-16,23,31}$ Other studies have identified that large nodules $(\geq 3.0 \mathrm{~cm})$ are not associated with any significant increase in false negative rate, while yet other studies have shown an even lower false negative rate compared with a nodule size of less than $3.0 \mathrm{~cm} .{ }^{18,21,29,30,32}$ In addition, a few studies have examined $4.0 \mathrm{~cm}$ as a cut-off point and have shown similar equivocal results between the two groups. ${ }^{11,13,14,20}$ In our study, the overall false negative rate was $5.4 \%$. When a cut-off point of $3.0 \mathrm{~cm}$ was used, the false negative rate in nodules less than $3.0 \mathrm{~cm}$ was $0 \%$ compared with $7.0 \%$ in those of $3.0 \mathrm{~cm}$ or greater. The cut-off size of $3 \mathrm{~cm}$ was chosen in this study because the majority of nodules of 3.0 $\mathrm{cm}$ or greater are subjected to thyroid surgery in our institution. This stems from earlier study by Meko et al indicating a high prevalence of malignancy in large nodules (defined as $\geq 3.0 \mathrm{~cm}$ ), which carry significantly higher false negative rates $\left(16.6 \%\right.$ vs $0 \%$ in the study). ${ }^{10}$ The results are criticised, however, because the authors did not use ultrasound guidance in their fine-needle aspiration biopsy, which is a standard of care today.

There are several potential explanations why larger nodules are associated with higher false negative rates. First, by virtue of their size, a complete sampling of large nodules is more difficult than in small nodules because of cytological heterogeneity within the nodules. ${ }^{13,15}$ This means that an island of cancer cells may coexist with a larger proportion of benign cells within the nodule. This risk may be reduced by taking multiple samples from different parts of the nodule, depending on its size, to obtain more representative results. Second, sampling error may contribute to the perceived higher false negative rates in large nodules. This might occur when cells taken for the sampling are not from the index nodule. Ultrasound guidance and on-site processing of the biopsy specimen are among the recommendations to improve sampling error. $^{19}$ Although the rates of malignancies between nodules less than $3.0 \mathrm{~cm}$ and those of $3.0 \mathrm{~cm}$ or greater are comparable, the false negative rate was higher in the 3.0 $\mathrm{cm}$ or greater group $(7.0 \%)$. It is therefore appropriate to repeat the fine-needle aspiration in this nodule size group, despite benign cytology, to improve diagnostic accuracy.

There were four false negative nodules (three papillary thyroid carcinoma and one follicular variant papillary thyroid carcinoma) in the $3.0 \mathrm{~cm}$ or greater group. The follicular variant of papillary thyroid carcinoma case was reviewed by a pathologist and met all the criteria for non-invasive follicular thyroid neoplasm with papillarylike nuclear feature. ${ }^{33}$ If this nodule was not considered definitely malignant, the corrected false negative rate was $4.0 \%$. When the cut-off of $3.0 \mathrm{~cm}$ was used, the false negative rate in nodules less than $3.0 \mathrm{~cm}$ remained $0 \%$ compared with $5.2 \%$ in those of $3.0 \mathrm{~cm}$ or greater.

We also evaluated the diagnostic accuracy and predictive value of ultrasound-guided fine-needle aspiration of thyroid nodules at a single institution. Our data demonstrate an overall sensitivity and specificity of $71.4 \%$ and $100 \%$, respectively. This is consistent with the reported sensitivity and specificity of fine-needle aspiration by Cross et al. ${ }^{9}$ The rate of non-diagnostic nodules (Thy1, Bethesda I) in our study was $8.5 \%$, within the recommended rate of less than $10 \% .^{34}$ The overall malignancy rate in nodules with benign cytology $(n=74)$ was $5.4 \%$.

Taken together, it remains challenging to interpret these conflicting results in the context of clinical application. A meta-analysis by Hammad et al involving 10,817 thyroid nodules reported that a nodule size of $3.0-5.9 \mathrm{~cm}$ had a greater risk of malignancy compared with a size of $3.0 \mathrm{~cm}$ or greater (OR 1.26)..$^{35}$ Interestingly, the author also reported that an even larger group of nodules (size $\geq 6.0$ $\mathrm{cm}$ ) is associated with a much lower risk of malignancy compared with a size of less than $3.0 \mathrm{~cm}$ (OR 0.84). However, this meta-analysis involved only four studies in each comparison, with one of the studies contributing more than $50 \%$ in weight to the overall data and therefore is a potential bias. Certainly, a larger meta-analysis involving all known studies done on this topic is warranted to elucidate current conflicting results.

\section{Conclusion}

Our data indicate that nodule size did not accurately predict the risk of thyroid malignancy irrespective of fineneedle aspiration cytology. Only fine-needle aspiration cytology was consistently reliable in determining whether or not a particular nodule was likely to be malignant or not. It remains difficult, therefore, to objectively justify routine diagnostic thyroid lobectomy solely because of a nodule size of $3.0 \mathrm{~cm}$ or greater.

\section{References}

1. Pellegriti G, Frasca F, Regalbuto C, Squatrito S, Vigneri R. Worldwide increasing incidence of thyroid cancer: update on epidemiology and risk factors. J Cancer Epidemiol 2013; 2013: 965212.

2. Siegel RL, Miller KD, Jemal A. Cancer statistics, 2015. Cancer J Clin 2015; 65: 5-29. 
3. Dean DS, Gharib H. Epidemiology of thyroid nodules. Best Pract Res Clin Endocrinol Metab 2008; 22: 901-911.

4. Alexander EK, Kennedy GC, Baloch ZW et al. preoperative diagnosis of benign thyroid nodules with indeterminate cytology. N Engl J Med 2012; 367: 705-715.

5. Hegedüs L. The thyroid nodule. N Eng/ J Med 2004; 351: 1,764-1,771.

6. Vecchia C La, Malvezzi M, Bosetti C et al. Thyroid cancer mortality and incidence: a global overview. Int J Cancer 2015; 136: 2,187-2,195.

7. McNally RJQ, Blakey K, James PW et al. Increasing incidence of thyroid cancer in Great Britain, 1976-2005: age-period-cohort analysis. Eur J Epidemiol 2012; 27: 615-622.

8. Haugen BR, Alexander EK, Bible KC et al. 2015 American Thyroid Association Management. Thyroid 2016; 26: 1-133.

9. Cross P, Chandra A, Giles T et al. Guidance on the Reporting of Thyroid Cytology Specimens, 2nd ed. London: Royal College of Pathologists; 2016.

10. Meko JB, Norton JA, Louis S. Large cystic/solid thyroid nodules: a potential false-negative fine-needle aspiration. Surgery 1995; 118: 996-1,004.

11. Carrillo JF, Frias-Mendivil M, Ochoa-Carrillo FJ, Ibarra M. Accuracy of fine-needle aspiration biopsy of the thyroid combined with an evaluation of clinical and radiologic factors. Otolaryngol Head Neck Surg 2000; 122: 917-921.

12. Albuja-Cruz MB, Goldfarb M, Gondek SS et al. Reliability of fine-needle aspiration for thyroid nodules greater than or equal to $4 \mathrm{~cm}$. J Surg Res 2016; 181: $6-10$

13. McCoy KL, Jabbour N, Ogilvie JB et al. The incidence of cancer and rate of false-negative cytology in thyroid nodules greater than or equal to $4 \mathrm{~cm}$ in size. Surgery 2007; 142: 837-844.

14. Wharry LI, Mccoy KL, Stang MT et al. Thyroid nodules $(\geq 4 \mathrm{~cm})$ : can ultrasound and cytology reliably exclude cancer? World J Surg 2014; 38: 614-621.

15. Mehanna R, Murphy M, Mccarthy J et al. False negatives in thyroid cytology: impact of large nodule size and follicular variant of papillary carcinoma. Laryngoscope 2013; 123: 1,305-1,309.

16. Giles WH, Maclellan RA, Gawande AA et al. False negative cytology in large thyroid nodules. Ann Surg Oncol 2015; 22: 152-157.

17. Pinchot $\mathrm{S}, \mathrm{Al}$-Wagih $\mathrm{H}$, Schaefer $\mathrm{S}$ et al. Accuracy of fine-needle aspiration biopsy for predicting neoplasm or carcinoma in thyroid nodules $4 \mathrm{~cm}$ or larger. Arch Surg 2009; 144: 649-655.

18. Porterfield JR, Grant CS, Dean DS, Thompson GB. Reliability of benign fine needle aspiration cytology of large thyroid nodules. Surgery 2006; 144 . 963-969.

19. Raj MD, Grodski S, Woodruff S et al. Diagnostic lobectomy is not routinely required to exclude malignancy in thyroid nodules greater than four centimetres. A N Z J Surg 2012; 82: 73-77.

20. Rosario PW, Salles DS, Bessa B, Purisch S. Low false-negative rate of cytology in thyroid nodules $\geq 4 \mathrm{~cm}$. Arq Bras Endocrinol Metab 2009; 53: $1,143-1,145$
21. Kuru B, Gulcelik NE, Gulcelik MA, Dincer H. The false-negative rate of fine-needle aspiration cytology for diagnosing thyroid carcinoma in thyroid nodules. Langenbeck's Arch Surg 2010; 395: 127-132.

22. Shrestha M, Crothers BA, Burch HB. The impact of thyroid nodule size on the risk of malignancy and accuracy of fine-needle aspiration: a 10-year study from a single institution. Thyroid 2012; 22: 1,251-1,256.

23. Bestepe N, Ozdemir D, Tam AA et al. Malignancy risk and false-negative rate of fine needle aspiration cytology in thyroid nodules. Surgery 2014; 160: 405-412.

24. Kamran SC, Marqusee E, Kim MI et al. Thyroid nodule size and prediction of cancer. J Clin Endocrinol Metab 2013; 98: 564-570.

25. Koo DH, Song K, Kwon $\mathrm{H}$ et al. Does tumor size influence the diagnostic accuracy of ultrasound-guided fine-needle aspiration cytology for thyroid nodules? Int J Endocrinol 2016; 2016: 1-6.

26. Shi H, Bobanga I, Mchenry CR. Are large thyroid nodules classified as benign on fine needle aspiration more likely to harbor cancer? Am J Surg 2017, 213: 464-466.

27. Godazandeh G, Kashi Z, Zargarnataj S et al. Evaluation of the relationship between thyroid nodule size with malignancy and accuracy of fine needle aspiration biopsy (FNAB). Acta Inform Med 2016; 24: 347-350.

28. Mchenry CR, Huh ES, Machekano RN. Is nodule size an independent predictor of thyroid malignancy? Surgery 2008; 144: 1,062-1,069.

29. Magister MJ, Chaikhoutdinov I, Schaefer $\mathrm{E}$ et al. Association of thyroid nodule size and bethesda class with rate of malignant disease. JAMA Otolaryngol Head Neck Surg 2016; 141: 1,089-1,095.

30. Ucler R, Usluogulları CA, Tam AA et al. The diagnostic accuracy of aspiration biopsy for thyroid nodules three centimeters or larger in size. Diagn Cytopathol 2015; 43: 622-628.

31. Cavallo A, Johnson DN, White MG et al. Thyroid nodule size at ultrasound as a predictor of malignancy and final pathologic size. Thyroid 2017; 27 : 641-650.

32. Tee YY, Lowe AJ, Brand CA, Judson RT. Fine-needle aspiration may miss a third of all malignancy in palpable thyroid nodules: a comprehensive literature review. Ann Surg 2007; 246: 714-720.

33. Nikiforov YE, Seethala RR, Tallini $G$ et al. Nomenclature revision for encapsulated follicular variant of papillary thyroid carcinoma: a paradigm shift to reduce overtreatment of indolent tumors. JAMA Oncol 2016; 2: 1,023-1,029.

34. Cibas ES, Ali SZ. The Bethesda system for reporting thyroid cytopathology. Thyroid 2009; 19: 1,159-1,165.

35. Hammad AY, Noureldine SI, Hu T et al. A meta-analysis examining the independent association between thyroid nodule size and malignancy. Gland Surg 2016; 5: 312-317. 